\title{
DUBÁI Y ABU DABI: ¿MITOS O REALIDAD?
}

\author{
Edgar Prada Rodríguez \\ Escuela de Negocios, Gestión y Sostenibilidad \\ Programa de Especialización en Gestión Empresarial \\ Especialista en Gestión Empresarial \\ pradaed@hotmail.com
}

Resumen

Hace ya algunos años hemos escuchando, en los diferentes medios, como redes sociales, periódicos, artículos e incluso en nuestros círculos sociales sobre el crecimiento de dos ciudades en los emiratos árabes unidos, todos los comentarios son diferentes, sin embargo lo único que tienen en común es que es una ciudad de mucho dinero, que tienen mucho petróleo y de ahí sus riquezas, donde se ven cosas que solo se encuentran en este sitio, que están las personas más adineradas del mundo y por ende los lujos, excentricidades y por qué no caprichos que solo estas personas pueden pagar, que tienen mascotas como jaguares, leones, camellos, tigres, etc. Que la policía patrulla sus calles en vehículos de alta gama como Ferraris, que los jeques tienen palacios enchapados en oro, que se movilizan en helicópteros para evitar los atascos en el tráfico, son solo algunos de los comentarios que personalmente había escuchado, también se dice que es un país extremadamente machista, que las mujeres no están al mismo nivel de los hombres, que deben cumplir una serie de compromisos y exigencias en la clandestinidad, que no tienen voz ni voto en ningún campo, ya sea laboral, político y mucho menos en el ámbito familiar, que deben permanecer ocultas bajo sus vestimentas porque no pueden exponer sus rostros ante los demás, que los hombres pueden tener las mujeres que deseen siempre y cuando este en la capacidad de cubrir los gastos de los diferentes matrimonios, estos y muchos más son las incógnitas que me lleve a este país y que incluso antes del viaje tuve la oportunidad de empezar a esclarecer. Pero no solo estos temas de tipo social fueron los que se tenían contemplados conocer, también se tenía unas visitas y charlas interesantes sobre proyectos que solo en este sitio del mundo han sido desarrollables y que al menos hoy son pioneros en su generación, para poder hablar de cada uno de estos temas quiero dividirlos e iniciar a abordarlos por separado, enfatizaré como se expresa en el título de este artículo en los emiratos de Dubai y Abu Dabi los cuales tuve la oportunidad de conocer.

\section{Organización y gobierno de los Emiratos Árabes Unidos}

Los EAU, están conformados por siete emiratos los cuales tienen su propio gobernante pero que a su vez tienen un solo presidente que los lidera y al cual todos rinden cuentas, los emiratos son, Abu Dabi, Dubái, Sharjah, Ajmán, Fujaira, Ras el Jaima y Um el Kaiwain, el emirato que lidera, donde está el presidente es Abu Dabi, desde su conformación se decidió que el gobernante de este emirato seria quien los representara 
en su máximo nivel, se escoge Abu Dabi por ser el emirato con mayor área, población y riqueza, tiene el $86.7 \%$ de área representados en más de $67.300 \mathrm{~km} 2$ del 100\% de los emiratos que tiene un área total de $77.699 \mathrm{~km} 2$, y una población de $39.4 \%$ respectivamente, (OFICINA DE INFORMACION DIPLOMATICA, 2016)este emirato aporta el presupuesto para mantener a los demás emiratos a excepción de Dubái y Sharjah que también manejan un amplio presupuesto propio generado de sus diferentes actividades económicas y que también aporta recursos para la mano tención de los demás emiratos, estos emiratos están a muy poca distancia el uno del otro, es así que en un solo día se podrían recorrer en transporte terrestre los siete emiratos, claro sin llegar a conocer realmente a fondo sus diferentes calles y costumbres. (Encyclopædia Britannica, Inc. , 2017)

\subsection{Actividad económica, Dubái, Abu Dabi}

Las dos ciudades hoy son epicentro de grandes construcciones y de empresas que están a la vanguardia en proyectos muy ambiciosos en los diferentes sectores financieros, Dubái concentra gran parte de su economía en la apertura de empresas internacionales, firmas muy importantes a nivel mundial que ya hoy tienen presencia en esta ciudad y que desde aquí están trabajando en mostrar todo lo nuevo y de gran tamaño como lo es todo en estas ciudades, (euronews, 2016) tiene el aeropuerto más importante del medio oriente con conexiones a todo Europa, maneja la mayor afluencia no solo de pasajeros sino de mercancías del mundo, cuenta con la aerolínea Emirates, quienes se ha preocupado por tener uno de los mejores servicios a nivel mundial, próximamente operara el vuelo directo más largo del mundo conectando ciudad de Panamá y Dubái en un vuelo de 17 horas y 35 minutos, estableciendo un nuevo record en el mundo aéreo (BBC, 2017). Dubái es el emirato más abierto al mundo internacional, no solo por sus inversiones si no por brindar a los extranjeros espacios más abiertos, es una ciudad que le está apuntando al turismo y que se ha venido preparando a lo largo de estos años para recibir la afluencia que hoy maneja y que espera superar, (hosteltur, 2016)cuenta con centros comerciales con la presencia de las principales firmas a nivel mundial donde se puede encontrar lo que se requiera para cualquier extranjero y local, tiene espacios alrededor de la ciudad diseñados para escapar si así se quiere de la vida y presencia de locales como se refieren a los ciudadanos árabes, espacios donde se puede departir con familia y amigos de las diferentes actividades, comida y bebida que no es muy fácil poder encontrar en el común de los negocios de la ciudad, (Vero4travel, 2013)sin duda se ha pensado en todo para brindar confort y hacer que se torne acogedor la permanencia en esta ciudad tan lejana, recuerden que no solo estamos hablando de personas que realizan este viaje de turismo, también encontramos todas las personas que están desarrollando una actividad laboral en las grandes empresas que mencione ya están situadas con sus grandes firmas y generación de empleo en este emirato, pues si bien se puede transitar, comer, vestir libremente por toda la ciudad se debe respetar una cultura que no ve con los mismos ojos diferentes comportamientos, usos de vestido, formas de expresión e interrelación de personas del extranjero. (Riesgo País y Estudios Económicos, 2014) 
Dubái cuenta con el edifico más alto del mundo el cual es insignia de la ciudad, (CNNEspañol.com, 2014) este edificio ha sido un impulsador desde su planificación hasta su construcción y entrega final, sol006F en la realización de la obra se generaron más de 12000 empleos directos, brindando no solo participación a los locales sino que también trajo gente de más de 30 países para hacerlos participes del magno proyecto, cuando hablamos de los emiratos siempre nos referiremos con palabras como el más grande, el más alto, el de más afluencia, el más lujoso, son los adjetivos que caracterizan todas las iniciativas que se generan en ciudades como Dubai y Abu Dabi.

Abu Dabi es un emirato que ha basado su economía en la explotación petrolera, (wordpress, 2015)tienen una de las más grandes reservas a nivel mundial, sin embargo también se mueven en el sector de la construcción, la industria y la presencia de servicios financieros, tiene las construcciones más majestuosas lo cual se aprovecha para atraer turismo, visitantes de todo el mundo, especialmente de Europa, que siempre estarán dispuestos a gastar dinero para vivir la experiencia que esta ciudad ofrece, ya también es conocida por el mundo cinematográfico por sus espectaculares filmaciones de escenas únicas de este paisaje, otra de sus principales atracciones es la edificación de la mezquita Sheikh Zayed es la más grande de los emiratos unidos, está abierta para las jornadas de oración, pero también se abren algunas de sus alas perimetrales para la visita de turistas, es un sitio de mucha historia, se deben seguir algunas recomendaciones puntuales para no entorpecer e incomodar las costumbres religiosas del lugar, cuando se inicia el recorrido de Abu Dabi realizando la visita a este lugar se empieza a dimensionar lo que se encontrara al interior de la ciudad, la edificación es de más de 20 hectáreas de área, con jardines, espacios abiertos y cerrados que juegan sin problema con toda la majestuosidad que se quería lograr con su construcción. (eluniversal, 2017)

$\mathrm{Al}$ ingresar a la ciudad se pueden observar los rascacielos imponentes desde cualquier punto, (El Confidencial, 2017) se puede hacer un recorrido por los alrededores de los grandes palacios de los jeques, donde también existen pequeñas muestras de lo ostentoso de su diario vivir, es una ciudad donde se están desarrollando proyectos importantes que buscan un plan B para la economía del país, donde está presupuestado que al 2030 ya no dependerán de la explotación de petróleos y que por el contrario se le apuntara al aprovechamiento de energías renovables utilizando recursos que tienen en la zona desértica que habitan, aquí es cuando aparecen proyectos tan ambiciosos como la ciudad Masdar. (gallastegui, 2016)

Masdar es una ciudad que busca ser auto sostenible, un proyecto que inicio en el 2006 y que cuando inició buscaba ser inaugurada en el 2016, sin embargo esta no se salvó de la crisis mundial y también fue afectada en su presupuesto, sin embargo hoy está avanzando a muy buen ritmo y la fecha que se tiene proyectada en un $100 \%$ es para el 2030 , una ciudad que obtendrá toda su energía de paneles solares aprovechando el principal recurso que tienen el sol, toda su construcción desde su diseño está pensada de tal forma que se utilizara la menor energía posible en su funcionamiento diario, (ecointeligencia, 2011) la ubicación de sus edificios está diseñada de tal forma que uno le brinde sombra natural al que tiene al lado y de esta forma haga más factible la circulación de personas por sus interiores, los materiales que se utilizan tienen la característica de ser reutilizados, al igual que se revisa que su producción se haya realizado con el menor consume de energía posible, al iniciar la construcción de esta ciudad lo primero que se pensó y se construyo fue un muro perimetral que permitiera bloquear las fuertes tormentas de arena del sector donde se tiene una gran presencia de las mismas, el sistema de ventilación para las casas y oficinas está pensado de tal forma que se puede 
aprovechar la circulación de aire y distribuirlo en los diferentes espacios, sin embargo hace un tiempo muchos daban este proyecto por muerto porque es una ciudad que no está siendo visitada por nadie y esto generaba gran incertidumbre sobre su futuro y posterior éxito, la inyección y compromiso de empresas reconocidas como SIEMENS, cuya sede ya está operando en los edificios con una ocupación de más de 800 empleados directos, edificio que ha se ha convertido en un hito para la organización por ser pionero. (Perez, 2015)

Personalmente me parece que es un proyecto que vale la pena no solo esperar a su terminación, ya deberíamos estar implementando técnicas de construcción de este y porque no copiando modelos, al final es un bien para todos, para nadie es un secreto el problema serio que estamos afrontando por culpa del hombre, destruyendo día a día el mundo con construcciones contaminantes, mal manejo de desechos, mal gastando los recursos naturales y demás, es algo que ya está trabajando Masdar y repito ya deberíamos estar imitando, el problema es que siempre queremos construcciones y proyectos a corto plazo donde el beneficio sea casi que de inmediato y el retorno de inversión en el menor tiempo posible, nunca pensamos a futuro, en que viene, en que será el negocio y los problemas en corto tiempo, por este tipo de pensamiento es que seguimos siendo catalogados como países del tercer mundo, no estamos a la vanguardia porque no queremos trabajar en ello, queremos solo explotar lo que ya está y que otros se preocupen por crear, lo más triste es que sabemos de la capacidad y talento que tenemos como Colombianos y que sin intensión de generalizar estamos siendo pasivos y porque no decirlo perezosos para generar soluciones, tenemos todos los recursos en nuestro país y de pronto por eso no creemos que estos son finitos, que no son perdurables en el tiempo, nunca me ha gustado hablar en contra de mi país y mucho menos de lo que somos, pero en estos temas es bueno hacer una reflexión un poco agresiva, países que no cuentan solo por dar un ejemplo con agua potable, que solo tienen agua de mar y esto no fue impedimento para pensar en grande y para decir que si era posible, hasta llegar a crear ciudades enteras en medio del desierto y atraer la vista del mundo hacia ellos, no se pensaron en lo que no tenían por el contrario se enfocaron en lo que contaban y lo explotaron al máximo, claro algunos podrían decir tienen todo el dinero y nosotros no contamos con esta clase de gobernantes que generan y piensan en un todo, pero eso es una discusión de otro ámbito al cual no quiero enfatizar porque sería una discusión saliéndonos del contexto que queremos tocar, solo dejo el interrogante por si algún lector quiere sacar provecho del mismo.

Para seguir con la línea de empresas que operan en esta ciudad, podemos hablar de IRENA que sus siglas resumen (Agencia Internacional de Energías Renovables), (IRENA, 2015) es una organización intergubernamental que brinda apoyo a los países que quieren explorar y trabajar con sistemas de energías renovables, todo en busca del uso sostenible de las energías, sus campos de acción son la energía solar, la hidroeléctrica, la geotérmica, bioenergía y la energía eólica, para este caso en Abu Dabi están trabajando y prestando todo su apoyo a los proyectos con energía solar, la ciudad Masdar funciona con un gran número de paneles solares ubicados en grandes extensiones de desierto dentro y fuera de la ciudad, pero desde allí están explorando y firmando diferentes acuerdos con países de todo el mundo que quieran apostarle a estos sistemas novedosos de operación, (Clare Saxon Ghauri, 2015) grata sorpresa cuando en medio de una charla que nos dio esta organización encontramos un colombiano trabajando como director de una de las áreas de esta organización, también al ver un estudiante Bogotano que está cursando su pasantía en diferentes campos de apoyo, fue un orgullo como colombiano ver compatriotas haciendo país en un lugar tan lejano. 
También nos enteramos que Colombia es miembro de IRENA desde el año 2015 y que este año hace parte del consejo para el periodo 2017-2018, (Cancillería de Colombia, 2017) cosa que nos motivó bastante, pero la pregunta es, que estamos haciendo para impulsar estos proyectos y cuáles de esos se están presentando ante esta organización para ser asesorados y con el acompañamiento de IRENA poder darle buen curso?, preguntamos sobre esto a las personas que realizaron la charla y nos informan que hasta hoy no se ha presentado avances pero que ya existen algunos acuerdos de palabra y compromisos adquiridos de alguna forma al hacerlos públicos ante la junta general hecha en Abu Dabi. (ElMundo, 2017)

Creo que esta información debería ser divulgada a nivel general, que los colombianos estemos enterados que existen organismos interesados en apoyar este tipo de proyectos en su parte ejecutoria, IRENA no participa ni abre licitaciones de ningún tipo, (reve, 2014) tampoco asigna recursos para ningún proyecto, lo que hace es el proceso de asesoría lo cual muchas veces es lo que hace falta para quitar el miedo a enfrentarse a estos sistemas de operación que no se conocen y que las empresa y personas no se atreven a iniciar, ni mucho menos enfrentar solos, estos sistemas de energía renovables estoy seguro funcionarían en cualquier lugar de nuestro país, por toda la riqueza natural que tenemos, pero aún más me pregunto si estas nos serian alternativas de primera mano para llevar servicios a departamentos que no cuentan por diferentes razones y que tendríamos que sentir un poco de vergüenza no tener suplidos, será esta la razón por la que no se adelantan estos acercamientos, departamentos como la Guajira, el Choco, Vaupés, Arauca, solo por nombrar algunos que hoy no cuentan con servicios de energía, agua potable, sitios donde no funciona un centro de salud por que no hay condiciones como operar, como tener unas medicinas refrigeradas, como garantizar poder conectar un aparato a un sistema eléctrico y poder porque no salvar una vida en lugares donde el primer puesto de salud puede estar a horas en lancha o sistema terrestre sin fácil acceso, no tengo las cifras de cuantas mujeres pierden a sus hijos al momento de parir por no tener las condiciones básicas para atender un parto, cuántos niños mueren de desnutrición y deshidratación por no tener agua potable, serian cifras interesantes de revisar y poner sobre la mesa para sustentar proyectos como los mencionados anteriormente con energías renovables. ( ProQuest LLC All rights reserved, 2009)

Esperemos que nuestro gobierno siga avanzando con estas iniciativas no quiero ser juzgador y de pronto sesgar la visión de un lector con apreciaciones de pronto equivocadas, no sabemos si esta vez la intensión sea real y podamos asignar recursos que son bastantes los que poseemos, para generar soluciones, porque no de paso quitarle estos caballitos de batalla en campañas políticas, que pasaría si desde el gobierno nacional se cubrieran estas necesidades, cuáles serían las promesas de nuestro políticos en sus diferentes campañas, todo sistema, proceso, proyecto, está sujeto a mejora, sería interesante ver personas proponiendo sobre algo que funciona, es fácil opinar y prometer sobre algo que a la luz no opera, no satisface, no llena las necesidades, porque cualquier persona lo puede palpar, nadie nos va a contestar que no le gustaría tener los servicios públicos en su hogar cuando hoy no cuenta con ellos, ningún habitante de un sector lejano le va a contestar que no le gustaría tener vías de acceso que facilitaran su diario vivir, a cuantos campesinos no les facilitaría poder procesar sus productos desde su finca y sacarlos al mercado sin la cantidad de intermediarios que tienen que lo único que hace es encarecer los productos para llevarlos al consumidor final en una ciudad, porque ni pensar sacarlos del país, si no tenemos las garantías para llegar a una capital, menos tendremos el apoyo para pensar en comercializar fuera del país nuestros propios productos, claro quedarían sin negocio las grandes compañías que son las que aportan el dinero 
a campañas políticas y demás, no me estoy desviando del tema principal que es nuestra visita a los emiratos árabes unidos, solo que me gusta dar una vista general al problema y me gusta dejar interrogantes, lo que quiero resumir es que si sacáramos provecho de estas organizaciones para satisfacer los servicios básicos de las comunidades se podrían asignar estos recursos al mejoramiento de otros temas que también hacen difícil el progreso en un país con tantos recursos naturales y económicos, señores gobierno piénselo, sonara irónico lo que voy a escribir, pero si el campesino saca más productos al mercado de pronto hasta le pueden cobrar más impuestos para las arcas que tenemos, ya sería solo tema de ver como se repartirían estas utilidades, pero no nos quedaríamos con el sin sabor de no haberlo intentado, de no haber probado que es cruzar una frontera y ver el mundo, que es esa palabra que a veces la dicen en las noticias, que a veces leemos en un periódico, que será eso de la globalización.

Por décadas los grandes escritores y pensadores han dejado ver que no existe nada más fácil de manejar que una comunidad desinformada, esto sí que lo han entendido los gobernantes de los diferentes países y es por eso que se bloquea cualquier posibilidad de precisamente eso, información, conocimiento, diferentes formas de ver el sistema y por ende de entenderlo, ojala se genere o nos pique ese bichito de la curiosidad, con este tipo de artículos, ojala llegue a lugares donde se necesite que son todos, porque a veces creemos que los que están fuera de las ciudades principales son los más atrasados, pero es sorprendente cuando incluso en la capital de nuestro país pensamos y seguimos sesgados al sistema que nos quieren hacer ver los que manejan los hilos de las diferentes ramas en nuestro país. $\mathrm{O}$ cuantas veces hemos pagado nuestro tren, metro o como lo quieran presentar, cuántas veces hemos pagado la descontaminación de nuestro rio Bogotá, bandera de campaña de cuantas campañas y personas elegidas una y otra vez, me sorprendió hablar con personas en los emiratos donde están de acuerdo que sus gobernantes sigan siendo los mismos, donde existen líderes que ya llevan más de 25 años guiando el futuro de su pueblo, claro la diferencia es que con solo salir a las calles y dar un vistazo de 360 grados se les puede dar la razón, no voy a decir que estos países no tienen problemas de desigualdad y demás, quizá sí, quizá cuando vamos en plan de turistas como es normal que pase, no nos muestren la parte oculta, puede ser, pero por lo menos no se siente uno en un sitio donde el tiempo ha pasado y su progreso no se ha quedado estancado, por favor preguntémonos, cuestionemos es la invitación que quiero dejar, de pronto en una de estas encontramos las soluciones y terminamos siendo inventores de algo que no estaba en el radar de nadie.

En cuanto a la serie de incógnitas que llevaba antes de viaje pude despejar la mayoría o al menos a que magnitud existen, no vi mascotas exóticas en carros de millonarios asomándose por la ventana, tampoco el dispensador de lingotes de oro en un centro comercial como si fuese un cajero automático o maquina dispensadora de snacks, tampoco vi policías en patrullas de lujo pero si percibí el respeto que se tiene por la autoridad, lo que sí puedo asegurar es que son una potencia, que hay mucho dinero, que tienen un profundo respeto por sus creencias, que si se hacen las cinco oraciones en el día para dar gracias a Dios por la vida, que si tienen hambre de comerse el mundo y darse a conocer aún más con sus mega proyectos y de paso ser el sitio atractivo número uno no solo por turismo si no por negocio e inversiones, por ultimo verifique algo que me dijo el embajador personalmente de los Emiratos Árabes Unidos en Bogota y es que las mujeres están ocupando cargos y espacios muy importantes en el sistema, son personas muy importantes en el ámbito familiar, toman decisiones y corrigen a sus hijos independientemente la edad que tengan, es así que para los hombres uno de los estandartes más importantes y de mayor respeto es la madre y su esposa, me disculpo si no llegue a abarcar 
todos los temas pero es una cultura y un país con mucho que contar, por eso los invito a que lo experimenten tomando parte de una frase muy colombiana, quien lo vive es quien lo puede dimensionar, por cierto, no vi ningún hombre con su harén. 


\section{Bibliografía}

ProQuest LLC All rights reserved. (30 de Junio de 2009). Sistema integrado de recursos para el aprendizaje y la investigacion. Obtenido de http://ucentralprimo.hosted.exlibrisgroup.com/primo_library/libweb/action/display.do?tabs=details Tab\&ct=display\&fn=search\&doc $=$ TN_proquest $468756548 \&$ ind $x=1 \&$ recIds=TN_pr oquest $468756548 \&$ recIdxs=0\&elementId=0\&renderMode=poppedOut\&displayMod $\mathrm{e}=$ full \&frbrVersion $=$

Encyclopædia Britannica, Inc. . (2017). SIRAI. Obtenido de http://ucentralprimo.hosted.exlibrisgroup.com/primo_library/libweb/action/display.do?tabs=details Tab\&ct=display\&fn=search\&doc $=$ TN_britnica31319\&indx $=1 \&$ recIds=TN_britnica3 $1319 \&$ recIdxs=0\&elementId=0\&renderMode=poppedOut $\&$ displayMode $=$ full $\&$ frbrV ersion $=\&$ frbg $=\& a$

Perez, O. (2015). SIRAI. UN FUTURO MEDIOAMBIENTAL INCIERTO PARA LOS EMIRATOS ÁRABES UNIDOS, 1-14 .

BBC. (06 de febrero de 2017). MUNDO. Obtenido de MUNDO: http://www.bbc.com/mundo/noticias/2015/08/150817_internacional_panama_dubai_ emirate_vuelo_ultralargo_ng

Cancillería de Colombia. (25 de mayo de 2017). Cancillería de Colombia. Obtenido de Cancillería de Colombia: https://www.cancilleria.gov.co/en/newsroom/news/colombia-fue-elegidavicepresidente-agencia-internacional-energias-renovables-irena

Carrington, D. (20 de abril de 2014). CNNEspañol.com. Obtenido de CNNEspañol.com: http://cnnespanol.cnn.com/2014/04/20/arabia-saudita-construira-el-edificio-mas-altodel-mundo-un-kilometro-de-altura/

Clare Saxon Ghauri. (03 de junio de 2015). c. Obtenido de Clare Saxon Ghauri: https://www.theclimategroup.org/news/irena-hq-greenest-office-building-uae

ecointeligencia. (22 de diciembre de 2011). ecointeligencia. Obtenido de ecointeligencia: https://www.ecointeligencia.com/2011/12/masdar-ecociudad-del-desierto/

Ediciones SM. (20 de Agosto de 2016). Obtenido de http://www.edicionessm.com.co/quienes-somos/?sec=1

El Confidencial. (14 de abril de 2017). El Confidencial. Obtenido de El Confidencial: http://www.elconfidencial.com/multimedia/album/cultura/2017-04-14/los-diezrascacielos-mas-altos-del-mundo_1108491\#0 
ElMundo. (30 de marzo de 2017). ElMundo.com. Obtenido de ElMundo.com:

http://www.elmundo.com/noticia/Colombia-asumira-vicepresidencia-de-Irena/49384

eluniversal. (18 de febrero de 2017). eluniversal. Obtenido de eluniversal:

http://www.eluniversal.com.mx/articulo/destinos/2017/02/18/las-siete-mezquitasmas-impresionantes-del-mundo

euronews. (12 de octubre de 2016). euronews. Obtenido de euronews: http://es.euronews.com/2016/10/12/dubai-se-convierte-en-el-centro-mundial-de-laeconomia-halal

gallastegui, I. (14 de enero de 2016). diariosur. Obtenido de diariosur: http://www.diariosur.es/sociedad/201601/13/masdar-city-20160113163430.html

hosteltur. (11 de febrero de 2016). hosteltur. Obtenido de hosteltur: https://www.hosteltur.lat/122440_dubai-alcanza-142-millones-turistas-2015-7america.html

IRENA. (03 de junio de 2015). irena.org. Obtenido de irena.org: http://www.irena.org/News/Description.aspx?NType=A\&mnu=cat\&PriMenuID=16 $\&$ CatID=84\&News_ID=411

OFICINA DE INFORMACION DIPLOMATICA. (Julio de 2016). FICHA PAIS. Obtenido de FICHA PAIS:

http://www.exteriores.gob.es/Documents/FichasPais/EMIRATOSARABESUNIDOS _FICHA\%20PAIS.pdf

Perez, O. (2015). SIRAI. UN FUTURO MEDIOAMBIENTAL INCIERTO PARA LOS EMIRATOS ÁRABES UNIDOS, 1-14 .

Reddy, M. (2007). Vendor managed inventory. journal of advance in management research $4,83-88$.

reve. (20 de enero de 2014). revista eólica y del vehiculo electrico. Obtenido de revista eólica y del vehiculo electrico: https://www.evwind.com/2014/01/20/la-agenciainternacional-de-energias-renovables-impulsara-el-desarrollo-de-nuevos-proyectos/ 
Riesgo País y Estudios Económicos. (07 de octubre de 2014). coface. Obtenido de coface: http://www.coface.es/Noticias-y-Publicaciones/Noticias/La-economia-de-EmiratosArabes-Unidos-crece-con-fuerza-gracias-una-efectiva-politica-de-diversificacion

Vero4travel. (23 de enero de 2013). Vero4travel. Obtenido de Vero4travel: http://www.vero4travel.com/2013/01/centros-comerciales-dubai-mall-skiacuario.html

wordpress. (02 de marzo de 2015). teamayn. Obtenido de teamayn:

https://teamayn.wordpress.com/2015/03/02/abu-dhabi-como-ejemplo-de-economia/ 